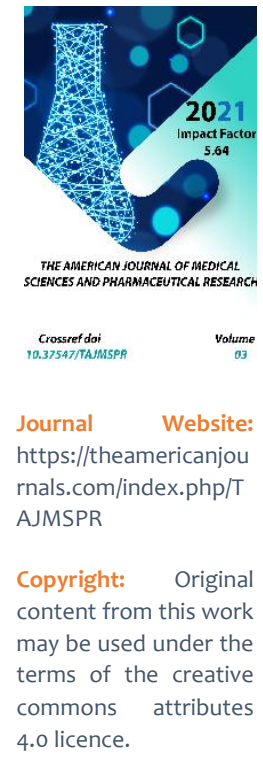

\title{
Modern Methods Of Acne And Post-Acne Therapy
}

Arifov S.S.

Center For The Development Of Professional Qualifications Of Medical Workers, Tashkent, Uzbekistan

Erkinlar Z.E.

Kasmed Medical Center Tashkent, Uzbekistan

Malikova N.N.

Republic Dermatovenerology Clinical Hospital; Tashkent State Dental Institute Tashkent, Uzbekistan

\section{ABSTRACT}

In the article, according to the data of foreign scientists and the authors' own observations, modern methods of topical therapy for acne and post-acne are presented.

\section{KEYWORDS}

Acne, Post-Acne, Treatment, Inadequate Therapy.

\section{INTRODUCTION}

Acne vulgaris (synonyms: acne, acne, acne) is a polymorphic multifactorial chronic disease of the sebaceous glands and hair follicles. Despite the fact that according to scientific studies, the prevalence of acne vulgaris (AC) among adolescents and young people reaches $80 \%$ or more, the disease can occur from infancy to old age (30). Severe forms of acne - conglobate, phlegmonous, papulopustular and nodularcystic, nodular - occur in $20-25 \%$ of patients. As a result of untimely or inadequate therapy, patients develop hyperpigmentation, atrophic or hypertrophic scars, milia, telangiectasias $(7,26)$. Currently, the Global Alliance for Acne
Treatment has proposed to combine these symptoms under the general term "postacne", which occurs in approximately $40 \%$ of patients with acne vulgaris (19). In our patients, post-acne symptoms were diagnosed in $63 \%$ of patients. Of these, $24.3 \%$ of patients had symptoms of mild severity, symptoms of moderate severity - in $65.7 \%$, severe symptoms were noted in $10 \%$ of patients. As can be seen from the data presented, the severity of postacne symptoms increases with the severity of the course of the disease (14). Clinical manifestations of acne and post-acne significantly reduce the quality of life of 
patients, lead to cosmetic defects and psychosocial maladjustment. $(2,4,5)$. The development of rational methods of therapy for acne and post-acne is an urgent task of dermatocosmetology.

\section{MATERIALS AND METHODS}

Modern approaches to the treatment of acne vulgaris have radically changed and the most rational approach is the use of drugs that affect all links in the pathogenesis of acne vulgaris, from hypersecretion of sebum to changes in its composition, follicular hyperkeratosis, reproduction of microorganisms, in particular P. acnes and inflammatory process in the focus (17). For patients with mild severity, it is sufficient to prescribe local therapy and/or combine it with cosmetic and physiotherapeutic procedures. The Global Alliance for Acne Treatment (2009) recommends early and effective treatment of acne to achieve a quick effect and to prevent and reduce the appearance of acne (15). According to the recommendations of the alliance, topical drugs should provide an effect on the maximum number of pathogenetic mechanisms of acne development, which is achieved through the use of a combination of two or three topical drugs. In many literary sources, topical retinoids, derivatives of vitamin $\mathrm{A}$ are considered the first barrier drugs in acne therapy, because they sufficiently affect the pathomechanism of acne and postacne, i.e. have comedolytic and antiinflammatory effects. Randomized, doubleblind and placebo-controlled studies have shown that they normalize follicular hyperkeratosis and hyperkeratinization and, therefore, should be recommended in the presence of microcomedones, comedones and to relieve inflammation in the focus, i.e. retinoids actually act on all links in the pathogenesis of acne $(16,21)$.

It was found that retinoids reduce the synthesis of procollagen by fibroblasts and inhibit the activity of matrix metalloproteinases. These mechanisms of action of retinoids are used not only to achieve a therapeutic effect but also to prevent postacne (26). In practice, retinoids such as adapalene, tretinoin and tazarotene are often used to treat acne. Clinical experience shows that topical retinoids reduce the number of comedones by $60 \%$, but the resolution of papules and pustules is slower (9). At the same time, $0.1 \%$ tazarotene is more effective than $0.1 \%$ adapalene, and even more effective than $0.025 \%$ tretinoin $(16,19)$. In a large number of patients with acne, the authors showed the effectiveness of $0.1 \%$ adapalene gel compared to $0.025 \%$ tretinoin cream (22). With a mild severity of acne, retinoids can be used as monotherapy, or they can be combined with other topical agents (benzoyl peroxide, topical antibiotics, azelaic acid, etc.). For moderate to severe acne, they are prescribed concurrently with systemic drugs. We have studied the effectiveness of Klinzit- $C$ (adapalene + clindamycin) in the treatment of acne. The results of the therapy showed that the drug quickly reduced the symptoms of acne and post-acne, due to its comedolytic and antiinflammatory effect (1). The action of benzoyl peroxide is directed against $P$. acnes and to date, there is no information in the literature on resistance to this agent. Benzoyl peroxide is used in the form of a shampoo, soap, lotion, cream, and gel and is given once or twice a day depending on the severity of the condition. The effect largely depends on the concentration and dosage form. For patients with sensitive skin, low concentrations (2.5-5\%) and water- 
based benzoyl peroxide are acceptable (15). A limited study (18) showed that the clinical efficacy of $10 \%$ benzoyl peroxide in resolving rashes and reducing free fatty acids is no better than $5 \%$ and $2.5 \%$ solutions. However, high doses are often irritating and dry skin. Topical antibiotics are effective against P. acnes and St. aureus, and also have anti-inflammatory effects. In practice, erythromycin and clindamycin are often used. Several studies have shown that antibiotics do not have a comedolytic effect $(11,12)$. Topical antibiotics are an alternative to oral and parenteral drugs and they have several advantages: ease of use, low risk of side effects, higher drug concentration in the infected area, low risk of bacterial resistance, and cost-effective. From local antibiotics in dermatology, $1 \%$ solution or gel of clindamycin, $2 \%$ cream, gel or lotion of erythromycin are often used. To prevent the development of antibiotic resistance, topical antibiotics as a monotherapy are not used $(7,9,11)$. The performed meta-analysis confirmed that clindamycin is one of the effective antibiotics for the resolution of inflammatory foci in patients with acne. The combination of clindamycin with benzoyl peroxide was much more effective than benzoyl peroxide monotherapy. The combination of these drugs reduces the risk of developing antibiotic resistance (23). Azelaic acid (20\%) is used for its antibacterial, antiinflammatory and comedolytic properties. The drug not only treats acne but also prevents the development of post-acne, i.e. it reduces the formation of pigmentation to one degree or another and brightens the skin $(19,21)$.

FDA (USA) in 2009 recommended topical application of $5 \%$ Dapsone gel ( 2 times a day) as an antimicrobial agent and for the treatment of inflammatory elements of acne. In clinical studies, it significantly reduced both inflammatory and non-inflammatory lesions at 4 and 12 weeks (21). A comparative study conducted over 12 weeks between women 18 years old and above and girls 12-17 years old showed that dapsone significantly reduced both inflammatory and non-inflammatory elements, while its clinical effect was more pronounced among adults, which indicates the appropriateness of its use. the drug in this category of persons (20). The combination of dapsone and retinoids has shown a very good effect in the treatment of comedonal acne. The authors believe that the action of the drugs is associated with the destruction of $\mathrm{P}$, acnes and anti-inflammatory action. The effectiveness of such combination therapy is more pronounced in women than in men and adolescents (28). To increase the effectiveness of acne therapy, prevent post-acne and reduce the risk of bacterial resistance, in recent years, combination drugs such as Klenzit $C$ (clindamycin + adapalene), Akania and Benzaclin (clindamycin + benzyl peroxide), benzamycin (erythromycin + benzoyl peroxide), zinerite have been used. (erythromycin + zinc acetate), Isotrexin (erythromycin + isotretinoin), Acnemycin plus, Erylik and Klinesfar (erythromycin + tretinoin) in the form of gels and solutions $(9,19,27)$. Elimination of acne symptoms is a difficult task, especially with cicatricial and atrophic processes. Since, along with acne symptoms, there are inflammatory elements of acne, this significantly reduces the choice of possible treatments. In the literature, there are no standards and recommendations for the treatment of post-acne scarring. (12.25).

Currently, the clinic uses surgical and nonsurgical methods to treat post-acne manifestations. Non-surgical methods of 
treatment are common in conservative cosmetology and they include hardware, injection, pharmacological methods, as well as a combination of the above methods $(6,24)$. Chemical peeling is a type of pharmacological method. As the author points out (8), the indications for its implementation are comedonal and papular-pustular forms of acne, as well as post-acne symptoms (atrophic scars and post-inflammatory hyperpigmentation). To correct superficial scars and dyschromia, medium-surface and medium peels are used using 30\% salicylic acid, $70 \%$ glycolic acid, $40-70 \%$ pyruvic acid, $15-39 \%$ trichloroacetic acids (5). The use of alphahydroxy acids for superficial chemical peels led to regression of hypo- and hyperpigmentation in $92.2 \%$ of cases, and pseudoatrophy in $54.4 \%$ of cases. Moreover, this acid did not affect cicatricial changes (3). To improve the quality of the skin of patients, hyaluronic acid is injected into the focus at a low concentration, which corrects atrophic scars in post-acne (17, 28). For the treatment of acne and post-acne, a number of authors in their works substantiate the advisability of using platelet-rich plasma (PRP). The clinical efficacy of platelet-rich plasma in atrophic skin conditions has been shown in several studies (27).

As a result of the use of PRP in the lesion focus, the generation of new stem cells occurs, which leads to the restoration of the dermis. In addition, under the influence of such therapy, the antioxidant system is activated, which saturates the tissues with oxygen. As a result, peeling of the epidermis decreases, the skin relief is levelled, wrinkles are smoothed, the intensity of hyperpigmentation is resolved (10). With post-acne, mesotherapy is widely used, which is carried out according to the principle of plasma lifting. Mesotherapy is used in the presence of comedones, cicatricial and atrophic elements, hyperpigmentation. In the form of microinjection, vitamins, enzymes, nucleic acids and amino acids, etc. are introduced into the focus. When mesotherapy is combined with other methods or drugs, good results can be achieved in the treatment of post-acne scars. (10.12). Often in post-acne therapy, mechanical dermabrasion is used to remove V-shaped and deep U-shaped scars. Since the scars are deep, the procedure is long, painful and can provoke the formation of keloid or hypertrophic scars, milia, persistent dyschromia. Microdermabrasion is considered to be more superficial and well tolerated by patients, but the results are not always satisfactory for patients. (12). To correct and improve stagnant spots and superficial atrophic scars, needling is used, i.e. multiple micro-perforations of the skin using a special sterile roller with needles no longer than $2 \mathrm{~mm}$. Studies have shown that the effectiveness of needling in the form of monotherapy reaches $25 \%$, which is comparable to the result of plasma lifting or mesotherapy $(6,12)$. The use of platelet-rich plasma in combination with the needling method increases the effectiveness of acne scar therapy (24).

Currently, the gold standard for the treatment of post-acne symptoms, especially atrophy and scarring, is the use of laser technology. For the treatment of cicatricial changes, classical ablation using a $\mathrm{CO}_{2}$ laser, an erbium Er: YAG laser with a wavelength of $1320 \mathrm{~nm}$, a diode laser with a wavelength of $1450 \mathrm{~nm}, \mathrm{RF}$ technology, as well as a fractional ablation method are effective $(10,22,23)$.

In the practice of a cosmetologist, ablative resurfacing with a $\mathrm{CO}_{2}$ laser is widely used. According to many authors, the mechanism of action of a $\mathrm{CO}_{2}$ laser on scar tissue consists in 
direct destruction or photothermolysis of the vessels of the microvasculature. This leads to suppression of inflammation and normalization of the architectonics of the scar (11). Clinical improvement of atrophic scars was observed in $50-80 \%$ of patients after the first procedure with a $\mathrm{CO}_{2}$ laser $(13,20)$. A good effect from the use of a $\mathrm{CO}_{2}$ laser was noted in other works $(15,21,22)$.

\section{CONCLUSION}

To study the effectiveness of methods of treatment of cicatricial and atrophic manifestations of post-acne, the authors divided 75 patients into 3 groups. The first group received an erbium Er: YAG laser for 6 months, the second group received 12 procedures for platelet-rich plasma and the third group received an erbium Er: YAG laser and 12 procedures for platelet-rich plasma. The results of the study showed that positive dynamics was noted in all groups. However, in patients of the third group, the clinical efficacy of therapy was better than in the first and second groups.

The above-mentioned information indicates that topical acne drugs contribute significantly to achieving a therapeutic effect in any form and severity of the disease and are an integral part of therapy.

Thus, timely initiated and rationally conducted therapy of acne vulgaris will contribute not only to the regression of the elements of the rash but to prevent the development of postacne symptoms and improve the quality of life of patients.

\section{REFERENCES}

1. Abdel-Hafez, K., Mahran, A. M., Hofny, E. R., Mohammed, K. A., Darweesh, A. M., \& Aal, A. A. (2009). The impact of acne vulgaris on the quality of life and psychologic status in patients from upper Egypt. International journal of dermatology, 48(3), 280-285.

2. Afra, T. P., Razmi, M., Narang, T., Dogra, S., \& Kumar, A. (2019). Topical tazarotene gel, $0.1 \%$, as a novel treatment approach for atrophic postacne scars: a randomized activecontrolled clinical trial. JAMA facial plastic surgery, 21(2), 125-132.

3. Boen, M., \& Jacob, C. (2019). A review and update of treatment options using the acne scar classification system. Dermatologic Surgery, 45(3), 411-422.

4. Brandstetter, A. J., \& Maibach, H. I. (2013). Topical dose justification: benzoyl peroxide concentrations. Journal of dermatological treatment, 24(4), 275-277.

5. Chim Ch. Acne Vulgaris. (2016). Dermatologic Care. ACSAP Book 2.

6. Del Rosso, J. Q., Kircik, L., \& Gallagher, C. J. (2015). Comparative efficacy and tolerability of dapsone $5 \%$ gel in adult versus adolescent females with acne vulgaris. The Journal of clinical and aesthetic dermatology, 8(1), 31.

7. Draelos, Z. D., Carter, E., Maloney, J. M., Elewski, B., Poulin, Y., Lynde, C., ... \& United States/Canada Dapsone Gel Study Group. (2007). Two randomized studies demonstrate the efficacy and safety of dapsone gel, $5 \%$ for the treatment of acne vulgaris. Journal of the American academy of dermatology, 56(3), 439-e1.

8. Dunlap, F. E., Mills, O. H., Tuley, M. R., Baker, M. D., \& Plott, R. T. (1998). Adapalene $0.1 \%$ gel for the treatment of acne vulgaris: its superiority compared to tretinoin $0.025 \%$ cream in skin 
tolerance and patient preference. The British journal of dermatology, 139, 1722.

9. El-Taieb, M. A., Ibrahim, H. M., Hegazy, E. M., Ibrahim, A. K., Gamal, A. M., \& Nada, E. A. (2019). Fractional erbiumYAG laser and platelet-rich plasma as single or combined treatment for atrophic acne scars: a randomized clinical trial. Dermatology and therapy, 9(4), 707-717.

10. Fabbrocini, G., Annunziata, M. C., D'arco, V., De Vita, V., Lodi, G., Mauriello, M. C., ... \& Monfrecola, G. (2010). Acne scars: pathogenesis, classification and treatment. Dermatology research and practice, 2010.

11. Fife, D. (2011). Practical evaluation and management of atrophic acne scars: tips for the general dermatologist. The Journal of clinical and aesthetic dermatology, 4(8), 50.

12. Fisher, G. J., Datta, S., Wang, Z., Li, X. Y., Quan, T., Chung, J. H., ... \& Voorhees, J. J. (2000). c-Jun-dependent inhibition of cutaneous procollagen transcription following ultraviolet irradiation is reversed by all-trans retinoic acid. The Journal of Clinical Investigation, 106(5), 663-670.

13. Greenhalgh, D. G. (1996). The role of growth factors in wound healing. Journal of Trauma and Acute Care Surgery, 41(1), 159-167.

14. Hasson A., Romero W.A. J. (2010). Drugs Dermatol. № 9 (12). P. 1507-1509.

15. Kircik, L. H. (2011). Efficacy and safety of azelaic acid (AzA) gel $15 \%$ in the treatment of post-inflammatory hyperpigmentation and acne: a 16week, baseline-controlled study.
Journal of drugs in dermatology: JDD, 10(6), 586-590.

16. Lynn, D. D., Umari, T., Dunnick, C. A., \& Dellavalle, R. P. (2016). The epidemiology of acne vulgaris in late adolescence. Adolescent health, medicine and therapeutics, 7, 13.

17. Mills Jr, O., Thornsberry, C., Cardin, C. W., Smiles, K. A., \& Leyden, J. J. (2002). Bacterial resistance and therapeutic outcome following three months of topical acne therapy with $2 \%$ erythromycin gel versus its vehicle. Acta dermato-venereologica, 82(4).

18. Pariser, D. M., Rich, P., Cook-Bolden, F. E., \& Korotzer, A. (2014). An aqueous gel fixed combination of clindamycin phosphate $1.2 \%$ and benzoyl peroxide $3.75 \%$ for the once-daily treatment of moderate to severe acne vulgaris. J Drugs Dermatol, 13(9), 1083-1089.

19. Seidler, E. M., \& Kimball, A. B. (2010). Meta-analysis comparing efficacy of benzoyl peroxide, clindamycin, benzoyl peroxide with salicylic acid, and combination benzoyl peroxide/clindamycin in acne. Journal of the American Academy of Dermatology, 63(1), 52-62.

20. Schoenberg, E., O'Connor, M., Wang, J. V., Yang, S., \& Saedi, N. (2020). Microneedling and PRP for acne scars: A new tool in our arsenal. Journal of cosmetic dermatology, 19(1), 112-114.

21. Song Y.X., Chen X., Tong T.X. (2020). $\mathrm{CO} 2$ dot-matrix laser combined with collagen dressing for facial acne scars. Pract Clin Inte Med, 20. pp. 153-5.

22. Sutaria, A.H., Masood S., Schlessinger, J. (2020). Acne vulgaris. StatPearls. [Internet] 
https://ecommons.aku.edu/pakistan_f hs_mc_med_intern_med/159.

23. Tan, A. U., Schlosser, B. J., \& Paller, A. S. (2018). A review of diagnosis and treatment of acne in adult female patients. International journal of women's dermatology, 4(2), 56-71.

24. Tanghetti, E., Harper, J. C., \& Oefelein, M. G. (2012). The efficacy and tolerability of dapsone $5 \%$ gel in female vs male patients with facial acne vulgaris: gender as a clinically relevant outcome variable. Journal of drugs in dermatology: JDD, 11(12), 1417-1421.

25. Thiboutot, D., Gollnick, H., Bettoli, V., Dréno, B., Kang, S., Leyden, J. J., ... \& Wolf Jr, J. (2009). New insights into the management of acne: an update from the Global Alliance to Improve Outcomes in Acne group. Journal of the American Academy of Dermatology, 60(5), S1-S50.

26. Tierney, E. P., Eisen, R. F., \& Hanke, C. W. (2011). Fractionated $\mathrm{CO} 2$ laser skin rejuvenation. Dermatologic therapy, 24(1), 41-53.

27. Zaenglein, A. L., Pathy, A. L., Schlosser, B. J., Alikhan, A., Baldwin, H. E., Berson, D. S., ... \& Bhushan, R. (2016). Guidelines of care for the management of acne vulgaris. Journal of the American Academy of Dermatology, 74(5), 945-973.

28. Zang, $H ., \quad \& \quad X u, Y . \quad N . \quad$ (2021). Effectiveness of $\mathrm{CO}_{2}$ laser therapy in treating acne depressed scar: A protocol of systematic review. Medicine, 100(3).

29. Zhang, D. D., Zhao, W. Y., Fang, Q. Q., Wang, Z. C., Wang, X. F., Zhang, M. X., ... \& Tan, W. Q. (2021). The efficacy of fractional $\mathrm{CO}_{2}$ laser in acne scar treatment: A meta-analysis. Dermatologic Therapy, 34(1), e14539.

30. Zang, H., \& Xu, Y. N. (2021). Effectiveness of $\mathrm{CO}_{2}$ laser therapy in treating acne depressed scar: A protocol of systematic review. Medicine, 100(3). 